\title{
Multilinguales
}

\section{Le ludique dans les manuels de français du cycle primaire : enjeux et perspectives}

The ludic in the french textbooks of the primary education cycle: stakes and prospects

\section{Sofiane Harrache}

\section{(2) OpenEdition}

\section{Journals}

Édition électronique

URL : https://journals.openedition.org/multilinguales/4407

DOI : 10.4000 /multilinguales.4407

ISSN : 2335-1853

Éditeur

Université Abderrahmane Mira - Bejaia

Référence électronique

Sofiane Harrache, «Le ludique dans les manuels de français du cycle primaire : enjeux et

perspectives », Multilinguales [En ligne], 12 | 2020, mis en ligne le 14 février 2020, consulté le 29 juin

2021. URL : http://journals.openedition.org/multilinguales/4407 ; DOI : https://doi.org/10.4000/

multilinguales. 4407

Ce document a été généré automatiquement le 29 juin 2021.

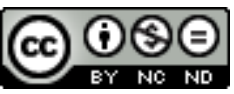

Multilinguales est mise à disposition selon les termes de la Licence Creative Commons Attribution -

Pas d'Utilisation Commerciale - Pas de Modification 4.0 International 


\section{Le ludique dans les manuels de français du cycle primaire : enjeux et perspectives}

The ludic in the french textbooks of the primary education cycle: stakes and prospects

Sofiane Harrache

1 La réforme éducative en Algérie, plus particulièrement dans le domaine de l'enseignement/apprentissage des langues, est accompagnée d'une restructuration des programmes en proposant de nouvelles orientations pédagogiques et des démarches d'enseignement/apprentissage axées davantage sur l'apprenant. L'institution scolaire s'est engagée à fournir des ressources pédagogiques, des moyens matériels et des outils didactiques élaborés en fonction des contenus pédagogiques des programmes afin d'aider les enseignants à renouveler leurs pratiques de classe et à dispenser un enseignement porteur de sens pour les apprenants. Par conséquent, de nouveaux manuels de français sont mis à la disposition des enseignants afin d'accompagner ces praticiens dans la mise en œuvre de ce projet et dans la conception, l'organisation et la réalisation de leur travail. Ces outils représentent une source quasi inépuisable d'inspiration qui accompagne les pratiques de classe de manière permanente.

2 L'analyse des documents officiels, plus particulièrement des contenus pédagogiques des programmes de français, montre la volonté de mettre en valeur la dimension ludique afin de rendre les situations d'apprentissage plus stimulantes et accessibles. Afin d'atteindre ces objectifs fixés, les concepteurs des programmes proposent une variété de supports comme :

Les textes à fonctions poétique et ludique: comptines, chansons, devinettes, poèmes, bandes dessinées (BD)... - Les textes qui induisent un échange verbal : dialogues, saynètes... - Les textes qui racontent : contes merveilleux, petits récits, BD, lettres ... (Ministère de L'Education Nationale, $2011: 27$ ). 
L'objectif est de sensibiliser les enseignants à l'importance de l'exploitation des jeux dans les pratiques pédagogiques. En fait, le recours au ludique représente une démarche pédagogique favorisant les processus d'apprentissage participatifs.

Notre travail s'est orienté vers l'analyse des activités ludiques proposées dans les manuels de français, il vise à élaborer des perspectives claires sur la conception et l'intégration du ludique en classe de langue au cycle primaire en tenant compte des orientations pédagogiques mises en œuvre et des changements opérés au niveau des dispositifs à adopter. Notre questionnement central est le suivant : dans quelle mesure les activités ludiques proposées dans les manuels de français du cycle primaire constituent-elles des outils didactiques et pédagogiques pouvant favoriser le développement des compétences disciplinaires et transversales requises dans les programmes officiels?

La présente recherche, de type exploratoire, se propose de développer quelques réflexions sur l'efficacité et l'impact des activités ludiques proposées dans les manuels de français sur les apprentissages des élèves. Nous nous efforçons d'expliquer les apports, les limites ainsi que les modalités de mise en œuvre de ces activités en tenant compte des principes de l'approche par compétences, des prescriptions des textes officiels et des intérêts de l'apprenant algérien.

\section{Le ludique, un outil d'apprentissage en classe de langue}

4 C'est grâce aux avancées de la psychologie, des sciences de l'éducation et de la didactique des langues étrangères que le ludique est reconnu comme étant un outil didactique incontournable dans les méthodes d'enseignement les plus en vogue. Pour Marie Musset et Rémi Thibert, c'est dans le cadre de »l'enseignement des langues vivantes que le jeu a le plus percé, notamment avec l'approche communicative, qui a mis sur le devant de la scène les jeux de rôle» (M. Musset et R. Thibert, 2009: 03). En s'appuyant sur des connaissances scientifiques, les didacticiens s'accordent pour donner une caution scientifique au jeu en soulignant son rôle dans la construction des connaissances des apprenants et le développement de leurs compétences disciplinaires et transversales.

Dans cette optique, les chercheurs s'interrogent sur l'efficacité de l'exploitation des activités ludiques en classe de langue et orientent leur réflexion vers les effets de cette exploitation non seulement sur l'ensemble des processus affectifs et cognitifs, mais aussi sur le développement langagier et social de l'apprenant, et sur sa réussite scolaire; selon Weiss, l'introduction des activités ludiques permet aux apprenants "d'agir et de réagir de façon adéquate dans les différentes situations de communication dans lesquelles ils pourront se trouver et dans lesquelles ils seront amenés à utiliser la langue étrangère » ( $\mathrm{F}$. Weiss, 2002 : 08).

En d'autres termes, ces activités permettent de créer des environnements cognitivement favorables à l'apprentissage des langues, elles favorisent l'accomplissement des tâches langagières et placent l'apprenant dans une position d'usager de la langue étrangère.

6 Les activités ludiques sont privilégiées par l'approche communicative. Néanmoins, introduire ces jeux en classe de langue est une affaire complexe, et comme l'affirme clairement Haydée Silva : 
«l'incontestable intérêt que suscite le jeu depuis une trentaine d'années reste toujours difficile à transposer de manière concrète par les enseignants. » (H. Silva, $2008: 22$ ).

Les enseignants de langue éprouvent des difficultés à transposer pratiquement ces nouvelles idées dans la réalité de la classe.

\section{Les enjeux des activités ludiques en didactique des langues-cultures}

7 L'exploitation du ludique en classe de langue peut se justifier par le fait que cet outil permet de faire évoluer les apprenants afin qu'ils puissent acquérir de nouveaux savoirs et de nouvelles règles. De plus, la pratique du ludique permet non seulement de garantir pleinement l'engagement des apprenants dans l'assimilation et le réinvestissement des connaissances dès le premier cycle mais aussi de développer leurs capacités d'analyse, de synthèse et d'application ; c'est pourquoi,

«tout enseignant devrait en injecter de larges doses dans son enseignement.»( $\mathrm{N}$.

Décuré, 2000 : 02).

Dans ce sens, le ludique aide à créer une ambiance plaisante en favorisant l'apprentissage dans l'action et la rencontre entre le maître et les apprenants.

Plusieurs auteurs plaident pour que l'on fasse du ludique un outil au service de l'apprentissage précoce d'une langue étrangère. Jean Vial accorde une place centrale au jeu dans son œuvre; pour lui :

« chez l'enfant (...), la parole est d'abord ludique, avant de devenir un instrument de

réquisition de l'adulte, de communication avec autrui. » (J. Vial, $1981: 34$ ).

Dans un univers ludique, le jeune apprenant a plus de capacités à reproduire des schémas et réalités phonologiques et accentuelles d'une langue étrangère et de s'exprimer avec moins de timidité. Le ludique permet à l'enfant d'inventer la réalité et de s'adapter rapidement à des environnements qui ne lui sont pas familiers. En d'autres termes, la pratique du ludique donne du sens aux apprentissages et favorise l'acquisition d'une méthode de travail et l'organisation de la pensée de l'apprenant tout en stimulant ses capacités de compréhension et de production.

Dans une classe de langue, le ludique est l'activité préférée des apprenants; il les rend plus curieux, il assure la création d'un climat propice à l'apprentissage et d'un environnement le plus authentique possible. Dans le même ordre d'idées, Grellet affirme que l'activité ludique constitue «le moment précis d'aider les enfants à s'adapter et s'intégrer, en les préparant à l'apprentissage tout au long de la vie.» (C. Grellet, 2000 : 07). Le jeu permet aux élèves d'entrer dans un univers linguistique et culturel différent de celui de leurs langues maternelles.

10 Le ludique constitue la pierre angulaire du grandiose édifice qu'est l'éducation; il est un ingrédient fondamental pour l'apprentissage précoce des langues. D'ailleurs, à cet âge, on cherche à

« développer l'intérêt de l'élève à partir de ce qu'il connait, on définit moins des objectifs de connaissance à maîtriser qu'une capacité d'apprendre à apprendre. » (JM de Queiroz, 2006 : 53).

Il s'agit d'un outil porteur de transformations et d'enrichissements personnels, il permet à l'apprenant d'agir sur son développement, de se construire un cerveau plus analytique, une personnalité compétente, un esprit synthétique, imaginatif et souvent créateur adapté aux conditions sociales. 
11 Le jeu est conçu comme une activité constructive du fait qu'elle permet à l'apprenant de se transformer lui-même, de pratiquer la langue étrangère hors du jeu et de se l'approprier ; il offre

« au pédagogue à la fois le moyen d'une meilleure connaissance de l'enfant et celui

d'un renouvellement des méthodes pédagogiques. » (UNESCO, 1979:05).

C'est dans le jeu que l'apprenant éprouve du plaisir et des sensations; il intériorise des rôles divers et comprend des situations. Dans ces contextes ludiques, l'apprenant apprend à coopérer et à accomplir des tâches langagières qui font sens pour lui ; il s'approprie les règles de groupe et intègre progressivement ses acquis. En d'autres termes, lorsque l'apprenant joue, il s'applique à acquérir le savoir et à le mettre ensuite en pratique.

\section{Méthodologie de l'étude}

12 Il est intéressant de déterminer la place accordée au ludique dans les trois manuels de français en tenant compte des objectifs fixés et des choix didactiques et méthodologiques opérés au niveau des programmes officiels. D'une part, nous tenons à examiner les modalités de mise en œuvre des activités ludiques et la nature des tâches à accomplir par les apprenants; nous nous efforçons de vérifier le respect des méthodes privilégiées actuellement par les concepteurs des programmes. D’autre part, nous cherchons à analyser ces activités du point de vue des éléments censés faciliter l'apprentissage et des démarches pédagogiques convoquées afin de favoriser le développement des compétences disciplinaires et transversales de l'apprenant.

\section{Analyse des activités ludiques proposées}

Nous classons et nous analysons les différentes activités ludiques proposées dans les trois manuels de français ( $3^{\mathrm{e}} \mathrm{AP}, 4^{\mathrm{e}} \mathrm{AP}$ et $\left.5^{\mathrm{e}} \mathrm{AP}\right)$. Ces livres ont été élaborés par des auteurs algériens et édités par l'Office National des Publications Scolaires (ONPS) en 2012. Nous tenons compte des contenus pédagogiques des programmes d'enseignement et des compétences de base à développer chez les jeunes apprenants. D'une part, nous nous efforçons de mieux cerner les démarches à adopter et les modalités d'exploitation des activités ludiques dans une perspective de rendre l'apprenant actif dans ses apprentissages. D'autre part, nous tenons à souligner les apports et les enjeux didactiques et pédagogiques relatifs à une telle exploitation.

\subsection{Les bandes dessinées : support fabriqué pour un dialogue authentique}

14 Cette catégorie d'activités ludiques repose en partie sur le principe d'une approche communicative selon lequel c'est en communiquant qu'on apprend à communiquer ; elle représente des moments d'inspiration et de communication orale tout en introduisant le verbal et le non verbal, la parole et le corps; et comme le dit Roux, la bande dessinée est

« un art constitué d'un ensemble de moyens d'expression qui lui sont propres - les bulles, les cases et les onomatopées - et des moyens dérivés du dessin, du cinéma et de la littérature.» (P. Roux, $1994: 25)$. 
Ces supports contribuent à la construction d'une culture commune aux apprenants dans un climat de respect et d'accueil de l'autre.

Les bandes dessinées tiennent une place importante dans le manuel de français de la $3^{\text {ème }}$ année primaire, elles sont liées aux actes de parole de chaque projet et regroupées sous la rubrique "J'écoute et je dialogue ». L'ensemble de ces activités représente des moments de réception et de production orale favorisant une mise en situation proche du réel et un apprentissage situé. Nous tenons à signaler que les contenus de ces activités sont proposés pour être lus à voix haute puis mimés et joués par l'enseignant et les apprenants. Ces supports fabriqués sont adaptés aux objectifs poursuivis et aux degrés de compréhension visés.

L'exploitation des bandes dessinées $(p .08,09)$ proposées dans la première séquence ( $M a$ nouvelle école) du premier projet vise, d'une part, à construire le sens d'un message oral en réception; d'autre part, elle permet aux apprenants de s'approprier l'emploi des mots et des expressions, et de maîtriser le système phonologique et les structures de la langue tout en accomplissant des actes de parole (présenter, se présenter).

Ces documents sont suivis par une série d'activités. En fait, c'est à partir de l'écoute, de la répétition, de la substitution, du transfert et du réemploi d'un certain nombre de mots et d'expressions que les apprenants peuvent s'exercer à la discrimination auditive et réaliser des actes de paroles (Bonjour! Je m'appelle..., Salut! Je suis..., Mon prénom est..., etc.) Dans ces activités, les apprenants sont invités à repérer et à faire la distinction entre les différents phonèmes de la langue française.

Les activités $(p .16,24)$ proposées dans la deuxième et de la troisième séquence (Mes camarades de classe / Mon métier d'élève) contribuent de manière importante à distinguer les différentes unités de sens, à repérer les noms propres et les noms communs, et à identifier les mots usuels et les mots outils. Dans ces situations, les apprenants peuvent apprendre à reconnaître des mots et des expressions, à saluer, à présenter quelqu'un et à se présenter (Bonjour les enfants! Au revoir, à demain! Voici deux nouveaux camarades! Je suis élève! J'ai un beau métier, Quel est ton sport préféré, etc.). C'est à partir de ces mots et expressions que le sens peut se construire. Ce sont les actes de parole sélectionnés dans le programme. Ces situations permettent aux apprenants d'enrichir leur vocabulaire en réutilisant leurs acquis dans d'autres situations.

Nous tenons à souligner la précieuse contribution des bandes dessinées proposées dans le deuxième projet. Ces activités peuvent susciter le goût de l'action collective en assurant l'implication des apprenants dans la construction des savoirs. C'est à travers l'activité (p. 32) proposée dans la première séquence (Je suis piéton) que les apprenants peuvent apprendre à demander ou à donner des informations (Je suis loin de..., Je suis près de..., La boulangerie est juste en face de l'école, etc.).

Les bandes dessinées $(p .40,48)$ proposées dans la deuxième et la troisième séquence de ce projet (Je suis passager, Je respecte le code de la route) favorisent l'apprentissage du système prosodique, de la musicalité et des intonations de la langue française. La première situation consiste à compléter et à répéter des énoncés de façon très simple afin d'amener les apprenants à imiter et à retrouver l'accent français (Quand tu te mets au soleil, porte un...! Quand tu manges, mets une...! Quand tu jardines, mets des...! Quand tu fais de la peinture, mets un...!). Dans la deuxième situation, les apprenants sont invités d'abord à lire quatre phrases de types différents afin de leur montrer les différentes intonations. Ensuite, ils complètent les énoncés en se basant sur des illustrations et en accompagnant leur intonation avec un geste particulier (Fodil, ne cours pas! Marche sur le 
trottoir. Que dois-tu mettre quand tu roules en voiture? Je roule à vélo. ). Enfin, les apprenants peuvent apprendre à faire des pauses en lisant des énoncés et incarnant les différents rôles des personnages (Nesma, mets ton casque. Oui papa, tout de suite. C'est plus sûr!). La dernière situation représente une occasion pour s'entraîner à maîtriser les rythmes de la chaîne parlée par le biais des activités de répétition et de la syllabation des phrases. Les apprenants sont invités à répéter la comptine (Quand je roule en voiture...) et à battre le rythme en frappant dans les mains. À travers ces situations, les élèves apprennent à affirmer et à donner des ordres (Je roule à vélo. Je mets la ceinture de sécurité. Mets ta ceinture! Ne cours pas! Marche sur le trottoir ! etc.).

Nous remarquons que les bandes dessinées ( $p .56)$ proposées dans la première séquence (Le coin vert) du troisième projet représentent une occasion privilégiée pour mettre en place des situations d'apprentissage suscitant l'intérêt des apprenants. Ces derniers peuvent repérer les différents actes de parole à partir de l'écoute de certains énoncés afin d'apprendre à demander ou à présenter des informations (Montre-moi les..., Voici le coin vert. Ne casse pas la tige! Il y a des plantes dans le coin vert.) A travers les situations introduites dans les bandes dessinées (p.64,72), les élèves apprennent à identifier non seulement les personnages et les interlocuteurs, leurs statuts et leurs répliques dans un dialogue mais aussi à mémoriser certains énoncés tout en réalisant des actes de parole (Tu veux aller au marché, Tu as pris ton panier? Quel est le fruit du chêne ?) Les images qui composent ces documents peuvent insuffler un nouvel élan en créant une véritable dynamique de groupe.

21 Les bandes dessinées (p. 80, 88, 96) proposées dans le dernier projet ont pour objectif de favoriser un apprentissage interdisciplinaire. Ces activités proposées abordent plusieurs domaines (environnement, éducation, santé, sport, etc.) à partir d'un seul thème : l'écologie, ce qui donne une cohésion aux situations d'apprentissage et aux tâches proposées. De plus, ces activités consistent à dire ce que font les enfants en s'appuyant sur des images, à identifier la consigne qui correspond à l'image et à pointer son doigt vers une pancarte, etc. Ces tâches ludiques sont proposées afin de retenir correctement des consignes (se laver les mains, brosser les dents, rincer la brosse, fermer le robinet, éteindre la lampe, jeter les papiers dans la poubelle, ramasser les bouteilles vides, etc.). L'accomplissement de ces tâches permet de donner du sens aux apprentissages liés aux disciplines et de développer des compétences nécessaires à la réalisation d'un projet pédagogique : "Confectionner un dépliant illustré sur la préservation de l'environnement pour l'afficher dans la classe ».

De plus, les activités qui accompagnent ces supports offrent des occasions uniques pouvant assurer le caractère transversal des apprentissages. En d'autres termes, la réalisation de ces tâches et actions permet à chaque apprenant de résoudre les problèmes (Se repérer dans le temps), de prendre la décision (Deviner les personnages des $\mathrm{BD}$ ), de mettre en œuvre sa pensée créatrice en racontant la suite de l'histoire, de coopérer avec ses camarades en leur demandant de protéger les plantes du coin vert, d'organiser et de communiquer les différentes informations contenues dans les images de façon appropriée.

Ces activités peuvent privilégier l'apprentissage actif et la communication orale en amenant les apprenants, d'une part, à interroger leurs camarades, à donner des informations et à repérer le thème et le cadre spatio-temporel; d'autre part, les apprenants peuvent apprendre à déduire un sentiment à partir d'une intonation et à interpréter un geste. Ces contextes représentent pour les apprenants des expériences 
pour développer des stratégies adéquates et leurs capacités à dialoguer et à échanger des idées.

\subsection{Atelier d'écriture ludique comme source de plaisir} maîtrise des gestes d'écriture en permettant aux élèves de reproduire progressivement des lettres, des mots, des phrases ou de courts textes de manière experte; selon Cuq, "l'atelier pédagogique fonctionne comme un lieu d'élaboration du savoir, de construction et d'interaction" ( J.-P. Cuq, 2003: 27). D'une part, ces situations renforcent l'ancrage des apprentissages dans une démarche concrète en permettant aux apprenants de faire appel à leur imagination et de s'exprimer librement. D'autre part, ces contextes incitent les élèves à produire des écrits, à agir et à interagir avec les autres participants de façon correcte et adaptée.

déclencheurs d'écriture permettant aux apprenants de transmettre des messages, d'identifier les caractéristiques propres à chaque type de texte et d'agir dans des échanges qui règlent leur vie scolaire et quotidienne. Selon Odette et Michel Neumayer, ces ateliers présentent de nombreux avantages en termes d'échange, de communication et de dialogue interculturel : "l'atelier est un lieu d'échanges: écrire en atelier, c'est mettre en circulation des écrits, des paroles, de l'expérience. » (O. Neumayer et M. Neumayer, 2008: 31 ). Pour réussir à atteindre les objectifs fixés, l'enseignant peut choisir la manière la plus efficace pour mener ses activités en utilisant l'ardoise ou le tableau, il peut opter progressivement pour un travail en équipe ou un travail individuel de sorte à établir un lien de confiance pour mieux travailler en atelier.

premier atelier d'écriture (p. 14) proposé dans la première séquence intitulée (Ma nouvelle école) vise à amener l'apprenant à réaliser un imagier thématique sur l'école tout en maitrisant le système graphique du français. À l'occasion de la rentrée des classes, l'apprenant se trouve dans une nouvelle école, plus belle et plus grande. Il s'agit, pour lui, d'une situation problème nouvelle, celle de présenter à sa sœur un imagier tout en décrivant cette école. En fait, l'apprenant peut consulter la boîte à outils afin de dessiner sa nouvelle école, la cantine et la bibliothèque sur une grande feuille en utilisant des couleurs. À la fin, l'apprenant recopie correctement sous son dessin la phrase suivante : Ma nouvelle école.

Dans le deuxième atelier (p. 22) proposé dans le cadre de la deuxième séquence intitulée (Mes camarades de classe), l'apprenant se trouve dans une situation complexe et nouvelle; celle de réaliser un fichier et de l'ajouter à l'imagier de l'école. À l'occasion de la visite du docteur, l'apprenant agit pour mettre à jour le fichier des prénoms des apprenants. D'abord, il place sous chaque photo la bonne étiquette du prénom sans oublier les nouveaux élèves. Ensuite, la tâche de l'apprenant est de lire à haute voix les prénoms en utilisant les présentateurs (c'est, voici, voilà). Enfin, la tâche de l'apprenant consiste à copier la phrase : Mes camarades de classe.

Dans le troisième atelier $(p .30)$, la situation problème proposée consiste à remettre au propre l'emploi du temps taché au moment de faire les devoirs à la maison par l'apprenant. L'activité est accompagnée d'un emploi de temps taché et d'une liste pour trouver les mots adéquats et compléter son emploi de temps. Avec ses camarades de classe, l'apprenant peut vérifier son travail et copier la phrase : Mon métier d'élève.

Multilinguales, 12 | 2020 
28 Afin de finaliser son imagier, l'apprenant peut rassembler les trois documents réalisés dans les trois séquences puis les présenter à ses camarades. Dans ces ateliers, les apprenants peuvent accomplir des tâches en respectant les règles du jeu: noter les noms des élèves, copier correctement les phrases, utiliser des crayons de couleurs, consulter la boite à outils, vérifier les erreurs d'orthographe avec ses camarades, etc. Ils peuvent mémoriser l'orthographe des mots fréquents et exprimer leurs opinions. Ces ateliers pédagogiques représentent des moments indispensables pour amener les apprenants à améliorer leur geste graphique, à agir dans des situations d'écriture que l'on qualifie d'authentiques et à développer leurs capacités d'autonomie.

L'objectif des ateliers proposés dans le cadre du deuxième projet consiste à réaliser une affiche illustrant des consignes de sécurité routière. Le premier atelier titré (Je suis piéton, $p .38$ ) a pour objectif d'apprendre aux apprenants à demander et à donner des informations. Cet atelier consiste à retrouver les mots effacés de la comptine en s'appuyant sur la boîte à outils. Par la suite, les apprenants peuvent lire les mots retrouvés tout en répétant le refrain (C'est plus sûr). À la fin de l'activité, chaque apprenant doit copier la comptine complétée en haut de l'affiche, il peut l'illustrer pour mieux présenter les consignes de sécurité routière.

Dans le cadre de l'atelier d'écriture ( $p$. 46) proposé à la fin de la deuxième séquence, l'enseignant invite ses apprenants à présenter des affiches et des dépliants dans le cadre de la journée nationale de la sécurité routière. D'abord, la tâche de chaque apprenant consiste à lever la main quand il entend une consigne de sécurité routière et à croiser les bras quand il entend d'autres consignes. Ensuite, il relie chaque consigne à une image, court et pointe son doigt vers une affiche accrochée au tableau tout en répétant à haute voix chaque consigne de sécurité routière. Enfin, il choisit une seule consigne, la copie sur son affiche et l'illustre par un dessin ou une image. L'accomplissement de ces tâches ludiques oblige les élèves à relever le défi et à oser se perdre. De plus, la stimulation provient de ces tâches ludiques à accomplir et de la motivation qu'elles suscitent. Cela nécessite non seulement un engagement personnel mais aussi l'acceptation de ses erreurs. Cette activité peut rendre les apprenants responsables et autonomes dans l'acquisition de nombreuses habiletés et compétences communicatives.

Le troisième atelier $(p$. 54) proposé dans la troisième séquence s'inscrit dans la continuité des deux ateliers précédents. Cette activité consiste d'abord à découper des panneaux et des consignes dans des revues, à mélanger ces découpages et à remettre ces découpages mélangés dans l'ordre. Ces tâches liées au plaisir de bouger et de jouer constituent une source de plaisir et de stimulation intellectuelle.

31 Ensuite, la tâche de l'apprenant consiste à faire correspondre chaque consigne à son panneau en s'appuyant sur les mots-clés de la boîte à outils. La tâche de l'enseignant consiste à afficher des panneaux, d'en enlever un à chaque fois que les apprenants referment les yeux, de demander aux apprenants de repérer le panneau en faisant un geste précis. La deuxième tâche consiste à demander aux apprenants sélectionnés de courir pour toucher le panneau correspondant à la consigne de sécurité routière lue par l'enseignant. Tout en captivant l'attention des participants, l'accomplissement de ces tâches ludiques favorise la compréhension des consignes et leur mémorisation.

Enfin, chaque apprenant doit coller le panneau et sa consigne sur l'affiche. Il propose un titre, organise son travail et présente l'affiche aux camarades. Cet atelier permet de 
développer une véritable pratique de l'écrit en amenant les apprenants à mémoriser les structures étudiées et à réinvestir leurs acquis de façon nouvelle et personnelle.

La production écrite libre est envisagée à partir du troisième projet. Le premier atelier (p. 62) proposé s'inscrit dans le cadre de la journée mondiale de l'arbre (le 21 mars); il consiste à réaliser la fiche technique d'un arbre fruitier. À cette occasion, les apprenants ont planté de jolies fleurs, mais quelques plantes du coin vert sont fanées. Face à ce problème et pour que les plantes ne se dessèchent pas durant l'absence des apprenants, ces derniers choisissent parmi les propositions présentées ce qui convient à une plante. Chaque apprenant dessine un cerisier sur une fiche cartonnée ; ensuite, il écrit le nom de l'arbre sur une étiquette et la colle à côté du dessin. Cet atelier permet aux apprenants d'accomplir des actions, de construire un univers de culture commune et de mettre en pratique leur vision écologique du monde.

Le deuxième atelier ( p. 70) vise d'abord à aider les apprenants à décrire oralement un fruit ou un légume (forme, couleur, goût, etc.). Cette étape est envisagée comme un espace de prise de risque en permettant aux apprenants de communiquer avec autrui de façon immédiate. La deuxième étape consiste à écrire la liste des courses de la semaine à venir (3 légumes, 2 fruits, 1 laitage, etc.). Dans la troisième étape, l'apprenant peut chercher dans la liste des fruits et légumes le nom d'un fruit, écrire son nom sur une autre étiquette et la coller sur la fiche cartonnée. Cet atelier vise à créer de petits espaces de discussion en langue française qui peuvent influencer les choix et les comportements linguistiques des apprenants.

Le dernier atelier (p. 78) consiste, d'une part, à lier chaque fiche à son illustration, à compléter la fiche (arbre-fruit) et à rédiger la fiche technique du cerisier; d'autre part, les apprenants sont invités à écrire la date du jour de l'exposition sur le cahier et à exposer la fiche technique du cerisier dans le coin vert de la classe. Tout en favorisant la communication avec les autres et la construction des liens d'amitié, cette activité peut garantir le développement des capacités de conceptualisation et l'autonomie des apprenants. L'ensemble de ces ateliers vise à assurer la consolidation des acquis des apprenants et la réalisation d'un coin vert dans la classe.

Dans l'atelier (p. 86) proposé dans la première séquence du dernier projet, l'apprenant confectionne un dépliant illustré sur la préservation de l'environnement; la première tâche consiste à choisir le bon titre parmi les deux titres proposés pour les trois phrases écrites au tableau. Ensuite, l'apprenant est invité à écrire le contraire des trois phrases et leur trouver un titre correspondant. Enfin, il recopie le titre et les phrases sur le dépliant. Ces ateliers représentent des faits de la vie, ils offrent aux apprenants l'occasion de donner du sens aux apprentissages et de développer plusieurs types de compétences langagières.

L'atelier d'écriture ( $p .94)$ proposé dans la deuxième séquence porte sur le thème de la préservation de l'environnement. D'une part, l'apprenant relie chaque image à la phrase qui lui correspond afin de rappeler les bons gestes; d'autre part, il dessine un tableau sur le dépliant et complète les phrases. En fait, c'est en pratiquant la langue, au cours de ces ateliers pédagogiques durant lesquels les apprenants rédigent des écrits et réalisent des tâches diverses, que les apprenants acquièrent des mécanismes linguistiques.

Dans le dernier atelier (p. 102) proposé dans le dernier projet portant sur la préservation de l'environnement, l'apprenant écrit le menu selon le modèle présenté ; 
il choisit le titre qui convient, le recopie sur le dépliant afin de le présenter aux camarades et l'afficher dans la classe. Cet atelier contribue à la création de nombreuses situations de parole en encourageant les apprenants à prendre le risque et à s'ouvrir aux autres. Il offre de véritables occasions d'utiliser la langue en situation en favorisant leur familiarisation avec un lexique approprié.

\subsection{Poèmes/chansons : environnements sonores adaptés aux rythmes d'apprentissage des apprenants} environnement sonore et authentique respectueux des rythmes d'apprentissage des apprenants; elles peuvent enrichir et faciliter non seulement les acquisitions fondamentales de l'apprenant mais aussi son autonomie. C'est pourquoi,

«aujourd'hui, on en fait un usage pédagogique et c'est surtout avec les mots des comptines que l'on joue. » (M. Tounsi, A. Bezaoucha et S. Guesmi, 2006:42). dans le but de permettre aux apprenants de lire et d'écrire des poèmes et des chansons tout en apprenant ce qu'est un vers, une rime et une strophe. Au total, il y a 15 poèmes et comptines dans ce manuel. Leur intégration dans le manuel scolaire vise à éveiller l'envie et le plaisir de lire et d'écrire pour explorer l'imaginaire et créer des textes poétiques.

Ces supports sont un excellent moyen pour travailler la prononciation. Durant ces activités, l'apprenant est appelé à montrer l'image accrochée au tableau et qui correspond à l'énoncé dicté par l'enseignant, à décrire un objet pour en faire deviner le nom, à compter le nombre de strophe et de syllabe en faisant un geste de la main et à trouver les lettres manquantes en pointant son doigt vers une affiche. Qu'il s'agisse de décomposer et de combiner les lettres d'un mot dans un ordre différent, de trouver le plus de mots possible qui riment pour rendre le vers cohérent en cinq minutes. Ces tâches ludiques relatives au plaisir de se déplacer et de jouer peuvent stimuler l'intérêt, l'imagination et les capacités intellectuelles des apprenants. Il est à noter que ces chansons et ces poèmes sont accompagnés d'illustrations.

Ces activités offrent d'excellentes occasions pour repérer et répéter les structures de la langue française. D’une part, les apprenants sont appelés à construire des phrases en se basant sur un modèle, à réécrire les phrases en petit poème, à compléter des phrases à trous par des homophones et à remettre les mots en ordre pour obtenir d'autres phrases. D'autre part, ils peuvent compléter une partie d'un mot, relier des mots, transformer une phrase et mettre le signe de ponctuation qui convient.

41 L'enjeu essentiel de la comptine (Donne-moi, p. 108) proposée dans la première séquence du troisième projet est d'inviter les apprenants à demander quelque chose, à demander la permission ou à demander le temps qu'il fait en réalisant dans une situation de communication orale des actes de parole pertinents. L'intégration de ce document vise à créer des ponts entre les cultures et à exposer les apprenants à une langue authentique en les sensibilisant à la prosodie. L'enseignant peut leur poser des questions afin de les amener à verbaliser la compréhension qu'ils se font du texte écouté.

Il convient de remarquer la présence d'une série de comptines (La pendule, La lune, L'eau, p.110) dans la première séquence du troisième projet. Ces activités sont introduites 
pour amener les apprenants à identifier un poème ou une comptine et à repérer les vers et les rimes. En d'autres termes, les apprenants peuvent apprendre à compter le nombre de vers qui composent une strophe et le nombre de syllabes qui composent le vers. La comptine (Saison d'automne, p. 114) constitue une occasion pour identifier les sons et mesurer le rythme dans un poème.

Les poèmes (La chanson du rayon de lune $p .122$, Les quatre éléments $p .126)$ visent à amener les apprenants à repérer les rimes qui reviennent dans ces poèmes et à retrouver les signes de ponctuation afin de marquer des pauses. L'activité (Le jeu des mots, p. 134) de C. Pierre est proposée afin d'apprendre aux apprenants à retrouver les mots qui sont formés à partir du même radical et les classer en deux familles.

Ces espaces de création permettent aux apprenants de jouer avec les mots et de former d'autres mots en changeant les syllabes et les lettres. Il s'agit d'extraire du texte les mots qui sont formés avec les mêmes lettres puis les classer en catégories. Ils doivent ensuite trouver le plus de mots possible dans chaque catégorie en décomposant les mots et en recombinant les lettres dans un ordre différent (mots de 2 lettres, de 3 lettres, de 4 lettres et de 5 lettres). Ainsi, les apprenants sont amenés à chasser l'intrus et trouver dans les séries proposées les mots qui ne sont pas formés avec le même radicale et les mots qui ne sont pas de la même famille.

\subsection{Devinettes, charades et mots croisés : un plaisir à la portée de tous}

Il faut noter que cette catégorie d'activités ludiques est peu représentée dans le manuel de français de la 4 ème année primaire. Le jeu de mots (p. 29) proposé dans le cadre de la première séquence du premier projet consiste à compléter le tableau des contes (Le petit chaperon rouge, Blanche neige, Cendrillon) ainsi que les éléments et les parties qui composent les contes (le héros, les actions, les lieux, les amis, les méchants, les objets magiques, la fin du conte). Cette activité permet de créer des récits à partir des éléments du tableau. L'enseignant peut diviser la classe en groupes de 6 et demander à chaque apprenant de remplir une rubrique sur une feuille. A l'aide des illustrations du tableau, chaque apprenant-joueur remplit sa rubrique, copie le texte sur un papier, le plie et le fait passer au suivant, etc. Cette forme d'écriture peut donner des récits passionnants, détaillés et cohérents. De la même manière, chaque apprenant peut chercher un livre de contes à la bibliothèque, choisir un conte, tracer le même tableau et compléter les éléments du conte choisi. Cette activité aide les apprenants à repérer les personnages et l'enchaînement des actions et à restituer la cohérence des textes déjà lus.

Le jeu de mots (p.43) vise à amener les apprenants à remettre en ordre les différentes parties qui composent le tableau et à relier chaque titre (la petite fille et les ours, pourquoi la mer est salée, le cordonnier riche) à son début et à sa fin. La mise en place de ces jeux de mots permet aux apprenants de s'écouter les uns les autres tout en utilisant la langue de façon naturelle dans des situations de communication de plus en plus authentiques. Dans le troisième jeu ( $p .59)$, la tâche de l'apprenant consiste à imaginer la fin de l'histoire en s'appuyant sur les éléments de l'Arc en ciel; il donne un titre à son histoire et lit l'histoire à ses camarades. Cette activité représente une excellente occasion d'apprentissage, car elle offre à l'apprenant la chance d'accéder à son imaginaire, de construire des stratégies individuelles de compréhension et de s'intéresser aux autres cultures. Ces activités concourent à développer les capacités des apprenants à 
s'exprimer, à communiquer et à résoudre des problèmes en exécutant des tâches facilement. Elles sollicitent la participation de tous les apprenants.

L'objectif du jeu (p. 74) proposé dans la première séquence du deuxième projet consiste à amener les élèves à trier et à classer les titres selon les rubriques du tableau (jeux, coloriages, bricolage, bandes dessinées, cuisine, histoires). Il s'agit d'une activité ludique à partir de laquelle il est demandé aux apprenants de compter précisément le nombre de titres dans chaque rubrique et de comparer les réponses données par chaque apprenant à celles des autres. L'enseignant peut accorder de l'importance aux propos des apprenants en les encourageant à prendre des décisions afin de progresser à leur rythme. Cette atmosphère aide les apprenants à résoudre des problèmes, à s'entendre avec les autres en acceptant les différentes façons de penser et à s'entraîner à des situations de la vie quotidienne.

Les jeux de mots $(p .89)$ proposés dans la deuxième séquence consistent à inscrire dans des cases les mots correspondant aux dessins. Les thèmes abordés appartiennent aux centres d'intérêt des apprenants. Ces derniers doivent retrouver dans la grille tous les mots de la liste. Ces jeux renforcent l'esprit d'ouverture culturelle des apprenants et l'apprentissage de l'altérité ; ils entraînent une plus grande implication des apprenants et un désir d'appartenance à un groupe. Le début et la fin de l'activité montrent le passage du monde réel au monde fictif.

47 L'exploitation de ces jeux suppose l'acceptation des règles et l'apprentissage par essais/ erreurs dans le cadre de l'approche ludique. Les apprenants-joueurs font des choix et agissent de manière stratégique pour atteindre les objectifs fixés. Ces jeux de mots représentent des espaces de créativité où les élèves éprouvent une jouissance à manipuler les mots; pour Sébastien Bailly, la devinette constitue "la première formalisation des jeux de mots, de ceux qui restent gravés dans la mémoire et qu'on n'oublie pas... » (Sébastien Bailly, $2004: 18$ ). Ces jeux suscitent la parole des apprenants en les sensibilisant à la dimension culturelle.

48 L'activité proposée dans la troisième séquence (p. 105) s'inscrit dans la rubrique "Charades et devinettes ». Dans cette activité, l'enjeu consiste à deviner la solution; les apprenants agissent pour se faire plaisir et pour réussir ou gagner en mobilisant leurs connaissances. Ils peuvent prendre des initiatives, élaborer leurs propres stratégies et développer leur imaginaire en construisant des fictions. Ces activités encouragent la prise de parole et l'interaction en classe. En somme, nous pouvons dire que les auteurs du manuel supposaient donc que ces jeux sont un moyen adéquat pour aider chaque apprenant à s'intégrer dans un groupe et à faire des choix réfléchis.

\subsection{Contes à oraliser : des moyens pour faire jouer un conte sous forme de théâtre}

Les contes occupent un grand espace dans le manuel de français de la $5^{\mathrm{e}}$ année primaire. Ces documents ont été choisis en adéquation avec les objectifs d'apprentissage afin de permettre aux apprenants d'écouter et de comprendre des messages oraux. Ces supports, riches sur le plan culturel, sont intégrés dans ce manuel en vue de permettre aux apprenants de s'approprier les différents types de discours et de développer leurs compétences de lecture de textes littéraires. Ils peuvent aussi apprendre à penser dans la langue cible en développant des automatismes liés aux techniques de structuration de phrases par le biais de l'accomplissement de ces tâches 
(réécrire le texte avec les signes de ponctuation qui manquent, remplacer les mots d'un texte par d'autres pour lui donner un sens, trouver les prépositions manquantes dans un texte, etc.). D'après Aron et Viala, il est important d'enseigner la littérature, car elle représente: "le vecteur d'une expérience esthétique, de l'adhésion à des valeurs, en même temps que de la transmission d'un savoir-faire argumentatif et expressif » (P. Aron et A. Viala, $2005: 3)$.

Le conte proposé dans le cadre du deuxième projet (Le crayon magique, p. 44) vise, d'une part, à amener les apprenants à identifier la structure narrative et les personnages de l'histoire. D'autre part, les apprenants peuvent repérer les trois situations qui composent l'histoire et les expressions qui introduisent chaque paragraphe. Dans le conte (Le petit coq noir p. 54), les élèves apprennent à identifier les particularités d'un conte et le héros de l'histoire; ils apprennent aussi à comprendre le rôle de chaque personnage dans l'histoire et à raconter en quelques phrases le conte étudié. Le troisième conte (C'était un loup si bête p. 64) consiste à identifier et à faire parler les personnages de l'histoire, et à jouer avec deux camarades les différents rôles.

La compréhension d'un texte suppose l'acquisition des connaissances et des savoir-faire particuliers. Les enseignants peuvent exploiter ces supports authentiques tout en proposant d'autres activités en fonction de l'ensemble de la séquence. Les questions et les activités de vocabulaire, de conjugaison et de grammaire proposées doivent permettre aux élèves d'apprendre à questionner ces textes et à développer des stratégies de lecture pour en saisir le sens, car préparer les apprenants à la maîtrise des textes, "c'est aussi leur fournir des instruments d'analyse qu'ils pourront réinvestir par la suite et les rendre autonomes. » (J.-P. Cuq et I. Gruca, 2005 :172.). Dans ces activités, l'enseignant peut inciter ses apprenants à développer leurs facultés de repérage tout en procédant par inférence. A partir de ces supports proposés, l'enseignant peut mettre en place plusieurs activités ludiques.

Lors de la phase de lecture, l'enseignant peut raconter ces contes sous une forme ludique en recourant à l'illustration, au ton de sa voix et à ses gestes pour aider les apprenants à comprendre l'histoire, à imaginer les personnages et à réagir. Durant cette étape, chaque apprenant est appelé à mimer les actions et à reproduire en temps réel les gestes de chaque personnage. Il peut identifier l'image/carte qui correspond à la séquence. Cette démarche ludique permet de faire de ces apprenants/auditeurs des spectateurs actifs et créatifs en sollicitant leur attention et leur imagination.

Durant la deuxième phase, l'enseignant peut inviter ses apprenants à transformer ou à modifier le contenu de ces histoires afin d'interpréter ces textes tout en incarnant les personnages de l'histoire. D'une part, il peut impliquer ses apprenants dans la mise en place de l'espace de jeu et dans la construction des règles ludiques pour que ces activités se déroulent dans des conditions favorables. D'autre part, il peut opter pour un travail en sous-groupes afin de donner la chance aux apprenants de prendre et de jouer les différents rôles. La mise en place de ces jeux de rôles permet de faire vivre les personnages et les scènes de ces contes.

\subsection{Le conte : vecteur de tous les apprentissages}

Il faut noter, dans le manuel de la $5^{\mathrm{e}}$ année primaire, la présence des textes fabriqués et des textes authentiques afin de permettre aux apprenants la construction des savoirs, des savoir-faire et des savoir-être. Avec patience, les enseignants peuvent confronter 
les apprenants à un genre littéraire bien précis et à des situations de communication de plus en plus riches et complexes. M. Lits témoigne de l'intérêt que représente la littérature pour les jeunes apprenants en disant : « si elle est, pour l'élève, découverte d'une écriture, d'un style, elle est aussi l'occasion d'un "grand jeu » expérientiel, d'une approche des grandes questions de l'existence. ( (M. Lits, 1994 : 26). Ces supports peuvent aider à la mise en place du processus d'apprentissage en offrant aux apprenants l'occasion de comprendre, de mémoriser, de raisonner et d'identifier des points de langue pour les réexploiter en lecture et en production écrite.

Nous avons relevé, en plus des textes narratifs cités précédemment, trois contes proposés dans le deuxième projet (Histoire de Babar, p. 45, Le chêne de l'ogre, p. 55, Suite et fin, p. 65). Ces supports visent à amener les apprenants à émettre des hypothèses de sens et à identifier les trois parties qui composent l'histoire (situation initiale, déroulement des événements, situation finale). De plus, les élèves apprennent à repérer les expressions qui introduisent chaque histoire et à identifier le cadre spatio-temporel, les personnages et les événements. Les apprenants apprennent à jouer la pièce tout en étant capables de reconstruire l'ordre chronologique des événements.

Ces textes narratifs sont utilisés comme outils de connaissances lexicales et encyclopédiques et un espace pour pratiquer les différentes formes de lecture et enrichir le lexique et le vocabulaire de l'apprenant. L'enseignant peut mettre en place des stratégies et encourager les apprenants à retourner au texte pour deviner le sens et maitriser la structure d'un texte narratif en établissant des liens entre les différentes parties. En fait, l'accomplissement de ces tâches permet aux apprenants d'acquérir une autonomie dans la lecture des textes.

Il est à noter aussi que la compréhension est l'objectif de la lecture. Les questions qui suivent ces supports sont ouvertes; elles accompagnent le texte, mais elles ne favorisent pas l'accès à l'implicite et ne permettent pas de faire un travail sur le contenu. Pour Gruca, les activités qui accompagnent les textes peuvent guider l'apprenant dans sa lecture ; c'est pourquoi, l'enseignant doit

« varier les procédures pour offrir différents angles d'approche et éviter d'utiliser en abondance les questions ouvertes pour permettre la réflexion.» (I. Gruca, 2009 : 178).

L'enseignant peut donner des consignes précises afin de permettre aux apprenants de réagir oralement et recueillir toutes les informations sur les différents personnages, leurs comportements, leurs traits physiques et psychologiques, etc.

En d'autres termes, cette phase peut offrir à l'apprenant la possibilité de dépasser la simple compréhension du texte en favorisant l'éveil de ses connaissances et la construction du sens; elle permet à l'enseignant de «conduire l'apprenant vers des points incontournables sur lesquels il l'incitera à ralentir sa lecture et à aiguiser sa réflexion." (I. Gruca, 2009 : 175). Les activités qui accompagnent ces textes ont été proposées afin de favoriser l'acquisition des notions fondamentales, la consolidation de certains apprentissages linguistiques et leur réinvestissement dans la production d'autres contes.

Il est intéressant de préciser que l'apport de ces textes dépend de leur usage pédagogique. En fait, les activités de langue qui accompagnent ces textes littéraires se font dans un cadre décontextualisé. Il s'agit d'un corpus constitué à partir des phrases tirées du texte lu, mais il n'y a pas de retour au texte de départ pour approfondir la compréhension de l'histoire. Pratiquement, les activités de conjugaison proposées ne 
permettent pas aux apprenants de mieux situer un fait dans l'histoire et de reconstituer l'ordre chronologique du conte. De même, les différentes phrases qui constituent essentiellement le corpus de base pour les activités de vocabulaire et de grammaire sont extraites du texte de départ, mais cela n'implique pas un retour à l'histoire et ne facilite pas la compréhension et l'interprétation des événements.

Les résultats de notre étude permettent de conclure que les supports et les documents proposés dans les trois manuels de français s'inscrivent, très généralement, dans la ligne des approches communicatives et dans le cadre des approches par compétences prônées par les textes officiels. L'introduction de ces documents peut servir à la mise en place de nombreuses activités et situations ludiques en classe de langue. Ces documents apparaissent comme une solution de remplacement aux pratiques traditionnelles garantissant la conciliation des contenus pédagogiques des programmes et des besoins des apprenants, des dimensions individuelles et sociales.

Ces activités s'inscrivent à l'intérieur d'actions sociales qui leur donnent une signification ; ce qui montre la volonté d'entrer dans la logique d'action. En fait, leurs avantages cités ci-dessus et leur importance dans le processus d'apprentissage incitent à la revalorisation de leur place dans l'acte pédagogique. Et pour retrouver le bonheur d'enseigner et d'apprendre, les auteurs des manuels préfèrent abandonner les choix monolithiques en proposant d'autres activités qui donnent aux apprenants l'efficacité et la flexibilité dans l'apprentissage de la langue.

Il est à remarquer que les activités ludiques proposées dans les trois manuels tiennent compte des contenus pédagogiques et des objectifs d'apprentissage et correspondent globalement à différentes phases d'apprentissage ; elles sont adaptées à la réalisation des tâches et placent les apprenants dans une position active. Dans cette perspective, et en fonction des spécificités des supports choisis, nous pouvons dire que les activités ludiques proposées sont adaptées aux exigences des approches méthodologiques nouvelles et tiennent compte des niveaux et des intérêts des apprenants.

Ces activités sont conçues dans une démarche pédagogique centrée sur la mise en place des apprentissages de façon progressive en tenant compte des dimensions communicatives et actionnelles. De ce point de vue, la pratique du ludique a pour but de créer des adaptations à des contextes sociaux dans lesquels l'apprenant, acteur social, a des tâches à accomplir et des choix à effectuer. En d'autres termes, nous remarquons que l'usage pédagogique privilégié dans l'exploitation des activités ludiques vise surtout à faire vivre la classe en tant que milieu social dans lequel l'apprenant mobilise stratégiquement les ressources dont il dispose afin de résoudre des situations problèmes.

Cette approche basée sur l'intégration du ludique en classe de langue constitue une véritable voie alternative pour faire un usage significatif de la langue française et pour une continuité dans les apprentissages. Globalement, les concepteurs des manuels ont adopté une démarche pédagogique cohérente qui donne du sens aux apprentissages et assure la compréhension et l'utilisation de la langue par les jeunes apprenants. Exposer les apprenants à des activités et situations ludiques leur permet de redécouvrir le plaisir d'apprendre, de retrouver la confiance en eux et de maîtriser les compétences de communication à l'oral et à l'écrit tout en développant leurs liens sociaux. 


\section{BIBLIOGRAPHIE}

ARON, P., et VIALA A., L'enseignement littéraire, Que sais-je ? Ed., Puf, Paris, 2005.

BAILLY, S., Jouez avec les mots, Ed. Eyrolles, 2004.

CUQ, J.-P. et GRUCA, I., 2005, Cours de didactique du français langue étrangère et seconde, Ed. PUG, Grenoble, 2005.

CUQ, J.-P., Dictionnaire de didactique du français langue étrangère et seconde, Ed. CLE International, Paris, 2003.

DE QUEIROZ, J-M., L'école et ses sociologies, Ed. Armand Colin, France, 2006.

DECURE, N., « Faites vos jeux », Recherche et Pratiques Pédagogiques en Langues de Spécialité : Cahiers de l'APLIUT, 2000, pp. 39-51. « Disponible sur https://hal.archives-ouvertes.fr/ hal-01300183/document » (Consulté le 05/01/2019).

GRELLET, C., Jeu entre la naissance et 7 ans, Un manuel pour les ludothécaires, Unesco, Paris, 2000. GRUCA, I., « Les enjeux de la littérature en didactique des langues-cultures : entre identité et altérité », La place de la littérature dans l'enseignement du FLE, Actes du colloque international, Université d'Athènes, Juin 2010.

LITS, M., Approche interculturelle et identité narrative. Littérature et cultures en situation didactique, Ed. ELA, n 93 (janvier-mars), 1994.

MINISTERE DE L'EDUCATION NATIONALE, Programme de français de la 3ªnnée primaire, Ed. O. N. P. S. Algérie, juin 2011.

MUSSET, M. et THIBERT R., » Quelles relations entre jeu et apprentissages à l'école ? Une question renouvelée ", Dossier d'actualité de la Veille scientifique et technologique, $\mathrm{N}^{\circ} 48$ octobre 2009. « Disponible sur http://veille-et-analyses.ens-lyon.fr/LettreVST/pdf/48octobre-2009.pdf » (Consulté le 02/01/2019).

NEUMAYER, O., et NEUMAYER M., Animer un atelier d'écriture, Ed. ESF, Issy-Les Moulineaux, 2008. ROUX, P., La BD, l'art d'en faire, Ed. CNBP, Poitoux Charentes, 1994.

SILVA, H., Le jeu en classe de langue, Ed. Pierre Carpentier, France, 2008.

TOUNSI, M., BEZAOUCHA, A. \& GUESMI, S., Guide du maître, français 3 primaires, Ed. O. N. P. S., Alger, 2006.

UNESCO, L'enfant et le jeu. Approches théoriques et applications pédagogiques, Coll. Etudes et documents d'éducation. N 34, UNESCO, France, 1979.

VIAL, J., Jeu et éducation, les ludothèques, Ed. PUF, France, 1981.

WEISS, F., Jouer, communiquer, apprendre, Ed. Hachette, Paris, 2002.

\section{RÉSUMÉS}

Dans le présent article, nous tentons d'analyser un corpus qui se compose des activités ludiques proposées dans les trois manuels de français afin de montrer l'importance de ces outils dans le développement des compétences des apprenants algériens. D’une part, nous cherchons à 
appréhender les représentations que les concepteurs de ces manuels se font de la dimension ludique dans l'enseignement/apprentissage du FLE. D'autre part, nous voulons déterminer la place accordée aux activités ludiques, l'impact ainsi que les perspectives de l'exploitation pédagogique du ludique en classe de langue. Nous tenons compte des exigences des approches pédagogiques et des objectifs assignés à l'enseignement du français en Algérie dans le cadre de la nouvelle réforme.

In this article, we try to analyze a corpus which is composed of the playful activities suggested in the three French textbooks in order to show the importance of these tools in the developement ok skills of algerian learners. On the one hand, we seek to apprehend the representations that the designers of these textbooks have of playful dimensions in the teaching/learning of the FFL. On the other hand, we want to determine the place granted to the playful activities, the impact as well as the prospects for the teaching exploitation for fun in language class. We take account of the requirements of the teaching approaches and the aims of the teaching of French in Algeria within the framework of the new reform.

\section{INDEX}

Keywords : playful activities, tale, writing workshop, poem, riddle, problem situation, task, communicative competence

Mots-clés : activités ludiques, conte, atelier d'écriture, poème, devinette, situation problème, tâche, compétence communicative

\section{AUTEUR}

\section{SOFIANE HARRACHE}

Université Kasdi Merbah Ouargla, Algérie 